\title{
Live birth rate comparison of three controlled ovarian stimulation protocols for in vitro fertilization-embryo transfer in patients with diminished ovarian reserve after endometrioma cystectomy: a retrospective study
}

Feiyan Zhao, Yonglian Lan, Tong Chen, Zhimin Xin, Yu Liang, Ying Li, Shuyu Wang, Jun Zhang ${ }^{*}$ and Xiaokui Yang ${ }^{*}$ (1)

\begin{abstract}
Background: Women with endometriosis and previous cystectomy may respond less well to gonadotropin stimulation, which results in fewer oocytes retrieved and poor pregnancy outcomes. Choosing an appropriate protocol for such populations is essential. This study involved an analysis of the effect of different controlled ovarian stimulation (COS) protocols on the clinical outcomes of in vitro fertilization-embryo transfer (IVF-ET) in women with diminished ovarian reserve (DOR) who underwent ovarian endometrioma cystectomy.

Methods: A total of 342 patients that underwent IVF-ET treatment at the Beijing Obstetrics and Gynecology Hospital from January 1, 2013 to April 30, 2018 were included in this retrospective study. The patients were distributed into three groups according to the COS protocols, namely prolonged $\mathrm{GnRH}$-agonist (Group A, $n=113$ ), GnRH-antagonist (Group $B, n=121$ ), and long $\mathrm{GnRH}$-agonist (Group $\mathrm{C}, n=108$ ). The clinical and laboratory parameters of the three protocols were analyzed and a logistic regression of clinical pregnancy and live births was conducted.
\end{abstract}

Results: There were no significant differences in the age, infertility duration, basic follicle stimulation hormone (FSH), luteinizing hormone $(\mathrm{LH})$, or estradiol $\left(E_{2}\right)$ levels as well as other baseline characteristics among groups $(P>0.05)$. The total gonadotrophin $(\mathrm{Gn})$ dosage and duration tended to be less in the $\mathrm{GnRH}$-antagonist group than in the others $(P<$ 0.05). No significant differences were found in the implantation rate and clinical pregnancy rate among the groups, but the prolonged GnRH-agonist group showed the highest rates. In addition, no significant differences were present in the number of retrieved oocytes, oocyte fertilization rate, embryo utilization rate, live birth rate, abortion rate, ectopic pregnancy rate, or multiple pregnancy rate in the three groups $(P>0.05)$. Age had a significant effect on both clinical pregnancy and live birth.

Conclusion: For those DOR patients who had undergone ovarian endometriosis cystectomy, the prolonged GnRHagonist protocol may achieve better clinical IVF-ET outcomes, but there were no significant differences from the other groups. The GnRH-antagonist protocol may reduce the cost and time of drug treatment. Age should be considered for its influence on pregnancy outcome. However, a larger sample size may be needed for further study.

Keywords: In vitro fertilization-embryo transfer, Live birth, Cystectomy, Diminished ovarian reserve, Endometrioma

\footnotetext{
*Correspondence: zhangjun9624@aliyun.com; xiaokuiyang2012@163.com

Department of Human Reproductive Medicine, Beijing Obstetrics and

Gynecology Hospital, Capital Medical University, 251 Yao jia yuan Road,

Chaoyang District, Beijing 100026, China
}

(c) The Author(s). 2020 Open Access This article is distributed under the terms of the Creative Commons Attribution 4.0 International License (http://creativecommons.org/licenses/by/4.0/), which permits unrestricted use, distribution, and

reproduction in any medium, provided you give appropriate credit to the original author(s) and the source, provide a link to the Creative Commons license, and indicate if changes were made. The Creative Commons Public Domain Dedication waiver (http://creativecommons.org/publicdomain/zero/1.0/) applies to the data made available in this article, unless otherwise stated. 


\section{Background}

Endometriosis is a common female disorder that occurs in about $10 \%$ of all women of reproductive age and $40-$ $50 \%$ of infertile women [1]. Due to the two-child per family policy in China and because more women are postponing childbirth due to pursuit of advanced degrees or developing a professional career, it is likely that infertility related to endometriosis will be encountered more frequently in China. Although endometriosis is a significant reason for progressive pelvic pain and infertility in married women of childbearing age, the etiology and pathogenesis remain unclear. Patients suffering from endometriosis could be adversely affected by distorted tubo-ovarian anatomy [2], triggering inflammation $[3,4]$ and oxidative damage $[5,6]$ and resulting in poor quality oocytes [7]. Nearly $17-44 \%$ of women with endometriosis have associated endometrioma [8]. Endometriosis involves not only formation of endometriomas, but also endometrial-like lesions in the organs outside of the uterus, for example leading to chocolate cysts or endometriomas inside ovary tissue. The European Society of Human Reproduction and Embryology (ESHRE) updated the guidelines about endometriosis in 2013. Laparoscopic ovarian cystectomy was proposed as the recommended surgical treatment for endometriomas $\geq 3 \mathrm{~cm}$ in diameter $[9,10]$.

Ovarian reserve is defined as the functional potential of the ovary and reflects the number and quality of the follicles in the ovary [11]. The sensitivity and specificity of ovarian reserve is equivalent to antral follicle count (AFC) and is actually more informative than folliclestimulating hormone (FSH), estradiol $\left(\mathrm{E}_{2}\right)$, luteinizing hormone ( $\mathrm{LH}), \mathrm{FSH} / \mathrm{LH}$ ratio or inhibin-B levels [12]. In recent years, some studies found that laparoscopic cystectomy for ovarian endometriomas may impair ovarian reserve by excessive and accidental removal of healthy ovarian tissue or compromising the ovarian vascular supply and reducing ovarian response to stimulation to some extent [13-18]. Other studies failed to prove its negative influence on ovarian reserve or response [1921]. Apart from AFC and basal hormone, anti-Müllerian hormone (AMH) level is also demonstrated to predict the magnitude of ovarian reserve as well as controlled ovarian stimulation (COS) responses [18, 22]. Endometrioma cystectomy, especially bilateral surgery, is deleterious with respect to ovarian reserve for $\mathrm{AMH}$, which decreased significantly based on a recent systematic review and meta-analysis and previous studies [12, 18, 23, 24].

Thus, there are conflicting and complex results regarding reproductive outcomes following cystectomy. This is particularly true for women undergoing assisted reproductive technology (ART) because women who underwent the cystectomy may respond less well to gonadotropin (Gn) stimulation and have fewer oocytes retrieved, which results in a higher cancelation rate and lower implantation and pregnancy rates for in vitro fertilization-embryo transfer (IVF-ET). Selecting a proper COS protocol is essential for this population and reproductive clinics should also consider previous surgery experience. Prolonged GnRH-agonist (prolonged $\mathrm{GnRH}-\mathrm{a})$ protocol, $\mathrm{GnRH}$-antagonist (GnRH-ant) protocol, and long GnRH-agonist (long GnRH-a) protocol are frequently used in women who underwent IVF treatment. We planned to determine whether these three protocols result in different pregnancy outcomes. In the present study, 342 IVF patients who suffered from diminished ovarian reserve (DOR) induced by endometrioma cystectomy were included and divided into three groups according to the different COS protocols. After IVF, we found that patients treated with the prolonged GnRH-a protocol had a higher implantation rate and clinical pregnancy rate than the $\mathrm{GnRH}$-ant group or the long GnRH-a group.

\section{Methods \\ Population}

All women who were referred to the study were selected according to IVF cycles performed in the Department of Human Reproductive Medicine, Beijing Obstetrics and Gynecology Hospital from January 1, 2013 to April 30, 2018. They routinely signed informed consent after a detailed explanation about some subsequent data collection for further study. Inclusion criteria included: age $\leq$ 40 years; duration of infertility > 12 months; women with a history of a previous laparotomic and/or laparoscopic surgery for unilateral or bilateral ovarian endometrio$\mathrm{ma}(\mathrm{s})$; and diagnosis of DOR including the following two or more features [25-27]: $10 \mathrm{IU} / \mathrm{L} \leq \mathrm{FSH}<25 \mathrm{IU} / \mathrm{L}$ or $\mathrm{FSH} / \mathrm{LH}>3$ on menstrual cycle day $2-3$, and AFC $\leq 5$. Patients were excluded if they had a diagnosis of polycystic ovary syndrome (PCOS), uterine fibroids, pelvic tuberculosis, autoimmune diseases, genital organ deformity, metabolic disorders, recurrent miscarriage, recurrent implantation failure, chromosomal abnormality, fertility caused by tubal factor or male factor, a history of other ovarian surgery, or received a steroid or immunosuppressant within the preceding 6 months. The study was approved by the Ethics Committee of the Beijing Obstetrics and Gynecology Hospital, Capital Medical University.

\section{Study design}

This is a retrospective study that assesses the data of 342 patients who received COS in a reproductive center. Excluding intracytoplasmic sperm injection (ICSI), we just included IVF cycles to minimize confounding factors from laboratory operations. These patients were divided 
into three groups according to different COS protocols: prolonged $\mathrm{GnRH}$-agonist protocol (Group A), GnRHantagonist protocol (Group B), or long GnRH-agonist protocol (Group C).

For the prolonged GnRH-a protocol (Group A), the following procedure was used. A standard full dose of triptorelin (Diphereline, $3.75 \mathrm{mg}$, Ipsen Pharma, France; or Decapeotyl, $3.75 \mathrm{mg}$, Ferring $\mathrm{GmbH}$, Germany) was administered in the early follicular phase for pituitary down-regulation. Down-regulation (no ovarian cysts $>8$ $\mathrm{mm} ; \mathrm{E}_{2}<50 \mathrm{pg} / \mathrm{ml}$ ) was confirmed after 28-42 days. Exogenous Gn (human menopausal gonadotropins, hMG, Livzon Group Livzon Pharmaceutical Factory, China or Highly Purified Menotrophin for Injection, 75 IU, Ferring $\mathrm{GmbH}$, Germany), generally $150 \mathrm{IU}$ to $300 \mathrm{IU} /$ day, was administered until the follicles reached maturity.

For the GnRH-ant protocol (Group B), exogenous Gn (Gonal-F, 75 IU, Merck Serono, Germany; or hMG, 75 IU, Livzon Group Livzon Pharmaceutical Factory, China; or Highly Purified Menotrophin for Injection, 75 IU, Ferring $\mathrm{GmbH}$, Germany), generally 225-300 IU/day, was administered at menstrual day 2-3 until the follicles reached maturity. Daily administration of GnRHantagonist (Cetrotide, $0.25 \mathrm{mg}$, QD Merck Serono, Germany) was initiated when Gn was used for six days or when the largest follicle reached a diameter of 13-14 $\mathrm{mm}$.

In the long GnRH-a protocol (Group C), a GnRHagonist (Decapeotyl, $0.1 \mathrm{mg}$, Ferring GmbH, Germany) was administered in the luteal phase of the previous cycle. Exogenous Gn (Gonal-F, 75 IU, Merck Serono, Germany; or hMG, 75 IU, Livzon Group Livzon Pharmaceutical Factory, China; or Highly Purified Menotrophin for Injection, $75 \mathrm{IU}$, Ferring GmbH, Germany) were used at doses ranging between $150 \mathrm{IU} /$ day and $450 \mathrm{IU} /$ day, generally in accordance with age, body mass index (BMI), basal FSH value, size and number of follicles, and $\mathrm{E}_{2}$ levels. Patients started serial transvaginal ultrasound from day 5 of ovarian stimulation and serum $\mathrm{LH}, \mathrm{E}_{2}$, and $\mathrm{P}$ measurements until the follicles reached maturity.

When the largest follicle reached $18 \mathrm{~mm}$ or at least two follicles reached $17 \mathrm{~mm}$ in diameter, human chorionic gonadotropin (hCG) $(250 \mu \mathrm{g}$, Merck Serono Inc., Geneva, Switzerland) was administered at night. Oocyte retrieval was conducted by transvaginal ultrasoundguided follicular puncture $36 \mathrm{~h}$ after hCG trigger. Luteal phase by daily progesterone (Progesterone capsules, 100 mg bid, Xianju Pharma, China and Progesterone soft capsules, $0.2 \mathrm{~g}$ tid, Besins Manufacturing, Belgium) was supported from the day of oocyte retrieval. Embryos were graded on day $2 / 3$ based on a ranking system that considered cell number, cell size, fragmentation, and multinucleation. Only one or two high-quality embryos were ultimately chosen to be transferred.
Pregnancies were diagnosed by positive serum hCG 14 days after embryo transfer. A clinical pregnancy was confirmed by visualization of a gestational sac on ultrasonographic examination 28-35 days after the embryo transfer. Luteal support treatment was continued until 10 weeks for confirmed pregnancies.

In the present study, we analyzed the outcomes including number of retrieved oocytes, fertilization rate, clinical pregnancy rate, spontaneous abortion rate, ectopic pregnancy rate, live birth rate, and multiple pregnancy rate in the three groups. Live birth was defined as one or more newborns with vital signs after $28 \mathrm{com}$ pleted weeks of gestation. Ectopic pregnancy was defined as a pregnancy not occurring inside the uterine cavity. Multiple pregnancy rate was defined as more than one fetus for a pregnancy. To adjust for confounding factors and evaluate baseline characteristics of patients recruited and type of stimulation protocol, we used logistic regression to investigate the potential elements influencing pregnancy and live birth.

\section{Statistical analysis}

Analyses of the data were performed using SPSS (Statistical Package for the Social Sciences) version 23.0 (IBM). Statistically significant differences among non-normally distributed data were determined using non-parametric Kruskal-Wallis $\mathrm{H}$ test, Chi-square test or Fisher's exact test to evaluate continuous or categorical variables and rates as appropriate. Logistic regression was performed for clinical pregnancy and live birth using the following variables: age, BMI, infertility duration, basal hormone levels, antral follicle count, and type of COS. All tests were bilateral and a $P$-value $<0.05$ was considered as statistically significant. Measurement results were expressed as medians (lower-upper quartiles) unless stated otherwise.

\section{Result}

\section{Baseline characteristics of the three groups}

During the study period, 342 patients undergoing cystectomy met the criteria and were included in this analysis. The patients' distribution according to their COS protocol was as follows: group A (113 patients, 33.04\%), group B (121 patients, 35.38\%), and group C (108 patients, $31.58 \%)$. Patient characteristics are summarized in Table 1. The three groups were similar in age, duration of infertility, BMI, basal FSH, basal $\mathrm{LH}$, basal $\mathrm{E}_{2}$, and AFC. There were no significant differences among the three groups $(P>0.05)$.

\section{Controlled ovarian stimulation parameters and IVF cycle characteristics}

Table 2 summarizes the characteristics of IVF cycles regarding the different COS parameters. Gn stimulation 
Table 1 General characteristics in patients with surgery-induced DOR treated with three controlled ovarian stimulation protocols

\begin{tabular}{lllll}
\hline & Group A $(\mathrm{n}=113)$ & Group B $(\mathrm{n}=121)$ & Group C $(\mathrm{n}=108)$ & $34.12 \pm 3.87$ \\
\hline Age (years) & $33.25 \pm 4.05$ & $34.19 \pm 3.90$ & $4.27 \pm 2.73$ & $\mathrm{NS}$ \\
Infertility duration (years) & $3.89 \pm 2.33$ & $4.40 \pm 3.07$ & $22.61 \pm 3.46$ & $\mathrm{NS}$ \\
BMI $\left(\mathrm{m} / \mathrm{kg}^{2}\right)$ & $22.53 \pm 3.93$ & $22.84 \pm 3.24$ & & $\mathrm{NS}$ \\
Type of infertility & & & $65(60.19 \%)$ & $\mathrm{NS}$ \\
$\quad$ Primary infertility & $68(60.18 \%)$ & $69(57.02 \%)$ & $43(39.81 \%)$ & - \\
$\quad$ Secondary infertility & $45(39.82 \%)$ & $52(42.98 \%)$ & $8.66 \pm 2.50$ & - \\
Basal FSH (IU/L) & $8.96 \pm 3.14$ & $9.30 \pm 3.18$ & $3.67 \pm 1.99$ & $\mathrm{NS}$ \\
Basal LH (IU/L) & $3.41 \pm 2.16$ & $3.69 \pm 1.78$ & $50.20 \pm 23.25$ & $\mathrm{NS}$ \\
Basal $\mathrm{E}_{2}$ (pg/ml) & $52.51 \pm 27.28$ & $55.29 \pm 32.90$ & $4.41 \pm 1.48$ & $\mathrm{NS}$ \\
Antral follicle counts & $4.14 \pm 1.43$ & $4.08 \pm 1.22$ & $\mathrm{NS}$
\end{tabular}

Abbreviations: $F S H$ follicle-stimulating hormone; $L H$ Luteinizing hormone; $E_{2}$ estradiol;

Statistical differences were calculated using the non-parametric Kruskal-Wallis $\mathrm{H}$ test, except for "type of fertilization" where the chi-square test was used Significant at $P<0.05$. Values are expressed as mean \pm standard deviation unless stated otherwise

duration was significantly longer in group A (12.90 \pm 5.22) compared with group $B(9.83 \pm 1.74)$ and group $C$ $(10.08 \pm 2.22)(P<0.05)$. There was a similar significance tendency in $\mathrm{Gn}$ dosage between group A (3493.75 \pm 1014.37) and groups B and C [(2581.61 \pm 827.11$)$ and (2594.24 \pm 1057.56 ), respectively, $P<0.05]$. Other parameters including hormone levels and thickness of endometrium on hCG day, number of oocytes retrieved, number of fertilized oocytes, and oocytes fertilized rate did not statistically differ among the three groups $(P>0.05)$.

\section{Reproductive outcomes of patients after IVF-ET}

The reproductive outcomes after IVF-ET treatment are presented in Table 3. The number of transferred embryos was determined in consideration of the conditions of all embryos together with patient preferences. Fresh embryo transfer cycles and total number of transferred embryos were assessed. In Groups A, B, and C, embryo utilization rate $(79.49,78.04,74.82 \%$, respectively), implantation rate (25.16, 18.01, 17.16\%, respectively), clinical pregnancy
(45.24, 33.33, 28.99\%, respectively), and live birth rate (32.14, 19.54, 24.64\%, respectively) were not significantly different $(P>0.05)$. Patients in group A appeared to have higher rates of implantation and pregnancy and were more likely to bear a healthy baby until delivery. The rates of spontaneous abortion $(28.95,34.48$, and $15.00 \%$, respectively), ectopic pregnancy $(0,6.90 \%$, and 0 , respectively) and multiple pregnancy $(5.26 \%, 0$, and $15.00 \%$, respectively) were not statistically different among Groups $\mathrm{A}, \mathrm{B}$, and $\mathrm{C}(P>0.05)$.

\section{Logistic regression of pregnancy outcomes in patients with surgery-induced DOR}

From the logistic regression (Table 4), we observed a significant effect of female age (OR $=0.881,95 \%$ CI $(0.799$, $0.963), P<0.05)$ on the clinical pregnancy. For live birth, age also appeared to be a significant variable $(\mathrm{OR}=$ 0.845 , 95\% CI $(0.764,0.934), P<0.05)$. Other baseline characteristics did not suggest any significant variation in the model.

Table 2 Controlled ovarian stimulation parameters and IVF cycles characteristics in patients with surgery-induced DOR

\begin{tabular}{|c|c|c|c|c|}
\hline & Group A & Group B & Group C & $P$ value \\
\hline Gonadotrophin duration (days) & $12.90 \pm 5.22^{\mathrm{a}}$ & $9.83 \pm 1.74^{b}$ & $10.08 \pm 2.22^{b}$ & $<0.001$ \\
\hline Gonadotrophin dosage (IU) & $3493.75 \pm 1014.37^{\mathrm{a}}$ & $2581.61 \pm 827.11^{\mathrm{b}}$ & $2594.24 \pm 1057.56^{b}$ & $<0.001$ \\
\hline Endometrial thickness on hCG day (mm) & $10.89 \pm 2.67$ & $10.55 \pm 1.94$ & $10.12 \pm 2.60$ & NS \\
\hline LH on hCG day (IU/L) & $2.15 \pm 1.60$ & $2.92 \pm 2.64$ & $2.60 \pm 2.62$ & NS \\
\hline $\mathrm{E}_{2}$ on hCG day $(\mathrm{pg} / \mathrm{ml})$ & $1713.34 \pm 1167.44$ & $1454.26 \pm 853.82$ & $1481.74 \pm 916.48$ & NS \\
\hline Progesterone on hCG day (ng/ml) & $0.97 \pm 1.16$ & $0.82 \pm 0.73$ & $1.02 \pm 1.53$ & NS \\
\hline Number of oocytes retrieved & $4.03 \pm 1.93$ & $3.67 \pm 1.92$ & $4.13 \pm 2.04$ & NS \\
\hline Number of fertilized oocytes & $3.11 \pm 1.63$ & $2.69 \pm 1.52$ & $3.00 \pm 1.46$ & NS \\
\hline Oocyte fertilization rate (\%) & $78.29 \pm 25.28$ & $73.52 \pm 28.92$ & $78.46 \pm 24.78$ & NS \\
\hline
\end{tabular}

Statistical differences were calculated using the non-parametric Kruskal-Wallis $\mathrm{H}$ test, except for "oocyte fertilization rate" where the chi-square test was used Significant at $P<0.05$. Values are expressed as mean \pm standard deviation unless stated otherwise

${ }^{a}$ Significantly different from group $B$ and group $C, P<0.001$ 
Table 3 Reproductive outcomes after IVF in patients with surgery-induced DOR

\begin{tabular}{|c|c|c|c|c|}
\hline & Group A & Group B & Group C & $P$ value \\
\hline Total cycles & 113 & 121 & 108 & - \\
\hline Fresh embryo transfer cycles & 84 & 87 & 69 & - \\
\hline Fresh transferred embryos & 159 & 161 & 134 & - \\
\hline Mean number of transferred embryos ddemembyos hello embryos embryos & 1.89 & 1.85 & 1.94 & - \\
\hline Embryo utilization rate $(\%, \mathrm{n})$ & $79.49(248)$ & $78.04(224)$ & $74.82(211)$ & NS \\
\hline Implantation rate $(\%, \mathrm{n})$ & $25.16(40)$ & $18.01(29)$ & $17.16(23)$ & NS \\
\hline Clinical pregnancy rate $(\%, n)$ & $45.24(38)$ & $33.33(29)$ & $28.99(20)$ & NS \\
\hline Live birth rate $(\%, n)$ & $32.14(27)$ & $19.54(17)$ & $24.64(17)$ & NS \\
\hline Spontaneous abortion rate $(\%, \mathrm{n})$ & $28.95(11)$ & $34.48(10)$ & $15.00(3)$ & NS \\
\hline Ectopic pregnancy rate $(\%, n)$ & 0 & $6.90(2)$ & 0 & NS \\
\hline Multiple pregnancy rate $(\%, n)$ & $5.26(2)$ & 0 & $15.00(3)$ & NS \\
\hline
\end{tabular}

Statistical differences were calculated using the Pearson's chi-square test or Fisher's exact test

Significant at $P<0.05$

\section{Discussion}

Surgical excision of an endometrioma has been the gold standard treatment though there is still a debate about it being detrimental to the ovarian reserve and ultimately associated with DOR [28, 29]. For infertile women after endometrioma cystectomy, spontaneous pregnancy rates may improve, however, IVF is still a primary option for patients still struggling to conceive [30-32]. During our practical clinic work, several protocols chosen for patients with endometrioma cystectomy that induced DOR were individually applied to COS. In addition, patients received the prolonged GnRH-a protocol, GnRH-ant protocol, or long $\mathrm{GnRH}$-a protocol according to personal conditions including menstruation, age, AFC, economic situation, and personal preference. In the present study, we found that clinical pregnancy rate and live birth rate in the prolonged GnRH-a protocol group was higher than the other two groups, but this difference was not significant.

Failed or diminished postsurgical ovarian reserve was reported after undergoing cystectomy for endometrioma [33]. The surgical removal of the cyst might be more

Table 4 Logistic regression of pregnancy outcome in patients with surgery induced DOR

\begin{tabular}{|c|c|c|c|c|c|c|c|}
\hline Baseline parameter & B & $\mathrm{SE}(b)$ & Wald $x^{2}$ & $P$ value & ORs & $95 \% \mathrm{Cl}$ & \\
\hline & & & & & & Lower & Upper \\
\hline \multicolumn{8}{|l|}{ Clinical pregnancy } \\
\hline Age & -0.127 & 0.046 & 7.513 & 0.006 & 0.881 & 0.799 & 0.963 \\
\hline BMI & 0.055 & 0.050 & 1.188 & 0.276 & 1.056 & 0.956 & 1.171 \\
\hline Infertility duration & 0.046 & 0.062 & 0.551 & 0.458 & 1.047 & 0.934 & 1.197 \\
\hline Basal FSH & -0.027 & 0.059 & 0.213 & 0.644 & 0.973 & 0.857 & 1.089 \\
\hline Basal LH & -0.018 & 0.084 & 0.047 & 0.828 & 0.982 & 0.834 & 1.169 \\
\hline Basal $E_{2}$ & -0.010 & 0.006 & 2.611 & 0.106 & 0.990 & 0.977 & 1.002 \\
\hline AFC & 0.139 & 0.126 & 1.232 & 0.267 & 1.150 & 0.930 & 1.546 \\
\hline \multicolumn{8}{|l|}{ Live birth } \\
\hline Age & -0.169 & 0.051 & 10.773 & 0.001 & 0.845 & 0.764 & 0.934 \\
\hline BMI & -0.017 & 0.049 & 0.113 & 0.736 & 0.984 & 0.893 & 1.083 \\
\hline Infertility duration & 0.054 & 0.067 & 0.655 & 0.418 & 1.056 & 0.926 & 1.203 \\
\hline Basal FSH & 0.064 & 0.054 & 1.392 & 0.238 & 1.066 & 0.959 & 1.186 \\
\hline Basal LH & -0.109 & 0.093 & 1.349 & 0.245 & 0.897 & 0.747 & 1.078 \\
\hline Basal $E_{2}$ & -0.007 & 0.007 & 1.055 & 0.304 & 0.993 & 0.980 & 1.006 \\
\hline AFC & 0.058 & 0.128 & 0.210 & 0.647 & 1.060 & 0.826 & 1.361 \\
\hline
\end{tabular}

$B$ regression coefficient; SE $(b)$ standard errors of regression coefficient; OR odds ratio

The modified Hosmer-Lemshow goodness of fit $X^{2}$ test statistics were $6.073(P=0.639)$ and $2.837(P=0.944)$, respectively 
catastrophic to certain parts of the vascular system and remaining healthy tissue around the cysts, otherwise an autoimmune reaction caused by a severe local inflammatory response could also harm the ovary function [29]. We observed that every cycle underwent one of the three COS protocols, and had a similar number of oocytes retrieved and oocytes fertilized. In our study, Group A and Group C both used GnRH-agonist, but differed in that patients in Group A usually got at least four-week pituitary down-regulation with one or two triptorelin injections. Over-suppression from long-term administration of the GnRH-agonists may cause poor ovarian response, thus requiring more $\mathrm{Gn}$ and taking more time. In Group B, patients endured fewer injections as well as a shorter treatment time, which may be a compromise for families in tough economic conditions. In all cases, the final pregnancy outcomes should be considered.

The embryo utilization rate, implantation rate, clinical pregnancy rate, and live birth rate in patients using prolonged $\mathrm{GnRH}$-agonist protocol were higher compared to the other two protocols. The protocol using long-acting GnRH-agonist may benefit the uterus by contributing to visible or invisible endometriotic lesion atrophy, thus providing an improved internal environment for implantation. Moreover, normal menstrual onset rarely occurs due to the overwhelmingly low level of estrin caused by the inhibition of the hypothalamic pituitary axis, the type of amenorrhea that can increase pinopodes and $\alpha v \beta 3$ integrin expression, which represents endometrial receptivity [34-36]. Furthermore, GnRH-agonist, especially for a long period, could evidently lower concentrations of various types of inflammatory cytokines, such as interleukin-1 and tumor necrosis factor, consequently reducing the intra-abdominal toxic effects on oocytes or embryos [37-39].

In our study, we found lower treatment time and pharmaceutical use in the GnRH-antagonist protocol. This protocol has therefore become widespread and is an indispensable part of ovarian stimulation in IVF due to lower risk of ovarian hyperstimulation syndrome (OHSS) and its function as preventing premature luteinization, which is essential for normal follicular development and oocyte maturation [40]. For pituitary desensitization, both daily administration of GnRHagonist and immediately blocking the secretion of $\mathrm{LH}$ with GnRH-antagonist can effectively block premature LH surges. Currently, in view of actual clinical practice, the GnRH-antagonist protocol has been gradually replacing the conventional long GnRH-agonist protocol, which was previously a major choice for all IVF patients.

Abortion rates were not significantly different among the three groups. Data in patients that used GnRHantagonist protocol gave a higher spontaneous abortion rate but with only a slight significant difference. The same result occurred for ectopic pregnancy rate and multiple pregnancy rate.

Aging was shown to have a negative effect on clinical pregnancy and live birth, as calculated by logistic regression. This finding is in agreement with previous studies showing that advancing age may increase oxidative stress as well as impair ovarian reserve and cytoplasmic quality due in part to decreased androgen levels [41]. Oxidative stress is related to increased granulosa cell apoptosis, which may account for inferior embryo quality and reduced live birth [42]. The condition of oxidative stress may be attributed to poor ovarian function, or have something to do with underlying psychological conditions $[43,44]$.

We note some deficiencies in the present study. First, its retrospective design inevitably failed to include all relevant data from the patient. Detailed operative recordings, such as time interval between surgery and COS, were not included in the study. Second, the embryo condition and pregnancy outcomes of frozenthawed embryo transfer (FET) of the included population was lacking, which probably had an influence on overall results. Third, the duration of $\mathrm{GnRH}$-agonist in the prolonged $\mathrm{GnRH}$-agonist protocol lacked subgroups that could be divided based on the specific dose and duration of pituitary suppression. Fourth, we measured the AFC and basal hormone level to roughly define DOR, but postoperative AMH levels were not measured; serum AMH levels may increase with the passage of time after surgery.

\section{Conclusions}

For those IVF-ET patients with DOR induced by ovarian endometrioma cystectomy, prolonged $\mathrm{GnRH}$-a protocol may be an optimal choice, however, the GnRH-ant protocol is less expensive and requires less time for drug treatment. Due to its effect on pregnancy and live birth, age is a significant factor that should not be overlooked in clinical practice. Further studies should use a larger sample size, due to the limited number of cases available, and include all relevant patient data for a comprehensive study.

\footnotetext{
Abbreviations

AFC: Antral follicle count; AMH: Anti-Müllerian hormone; ART: Assisted reproductive technology; BMI: Body mass index; COS: Controlled ovarian stimulation; DOR: Diminished ovarian reserve; $E_{2}$ : Estradiol; ESHRE: European Society of Human Reproduction and Embryology; FET: Frozen-thawed embryo transfer; FSH: Follicle stimulation hormone; Gn: Gonadotrophin; hCG: human chorionic gonadotropin; ICSI: Intracytoplasmic sperm injection; IVF-ET: In vitro fertilization-embryo transfer; LH: Luteinizing hormone; OHSS: Ovarian hyperstimulation syndrome; PCOS: Polycystic ovary syndrome
} 


\section{Authors' contributions}

Dr. Xiaokui Yang and Mr. Jun Zhang proposed the experimental design. Dr. Yonglian Lan, Dr. Zhimin Xin, Dr. Ying Li, Dr. Yu Liang and Mr. Tong Chen collected patients' information. Ms. Shuyu Wang and Mr. Jun Zhang checked the data of patients and verified the calculation and statistical approaches. Ms. Feiyan Zhao drafted the manuscript and constructed the tables. The author(s) approved the final version of this article.

\section{Funding}

1. Natural Science Foundation of China (81871133):

2. Beijing Municipal Administration of Hospitals Clinical Medicine Development (ZYLX201830)

\section{Availability of data and materials}

All data analyzed during this study are included in this article as tables.

\section{Ethics approval and consent to participate}

Ethical approval was obtained from the Ethics Committee of the Beijing Obstetrics and Gynecology Hospital, Capital Medical University.

\section{Consent for publication}

Not applicable.

\section{Competing interests}

All authors have declared that they have no competing interests.

Received: 10 June 2019 Accepted: 10 February 2020

Published online: 29 February 2020

\section{References}

1. De ZD, Borghese B, Chapron C. Endometriosis and infertility: pathophysiology and management. Lancet. 2010;376(9742):730-8.

2. Young VJ, Brown JK, Saunders PT, Horne AW. The role of the peritoneum in the pathogenesis of endometriosis. Hum Reprod Update. 2013;19(5):558-69.

3. Gazvani R, Templeton A. Peritoneal environment, cytokines and angiogenesis in the pathophysiology of endometriosis. Reproduction. 2002; 123(2):217-26

4. Harada T, Iwabe T, Terakawa N. Role of cytokines in endometriosis. Fertil Steril. 2001;76(1):1-10.

5. Matsuzaki S, Schubert B. Oxidative stress status in normal ovarian cortex surrounding ovarian endometriosis. Fertil Steril. 2010;93(7):2431-2.

6. Agarwal A, Apontemellado A, Premkumar BJ, Shaman A, Gupta S. The effects of oxidative stress on female reproduction: a review. Reprod Biol Endocrinol. 2012;10(1):49.

7. Gupta S, Agarwal A, Agarwal R, Loret DMJ. Impact of ovarian endometrioma on assisted reproduction outcomes. Reprod BioMed Online. 2006;13(3):349_ 60.

8. Busacca M, Vignali M. Ovarian endometriosis: from pathogenesis to surgical treatment. Curr Opin Obstet Gynecol. 2003;15(4):321.

9. Dunselman GA, Vermeulen N, Becker C, et al. ESHRE guideline: management of women with endometriosis. Hum Reprod. 2014;29(3):400-12.

10. Hwu YM, Wu SY, Li SH, Sun FJ, Lin MH, Lee KK. The impact of endometrioma and laparoscopic cystectomy on serum anti-Müllerian hormone levels. Reprod Biol Endocrinol. 2011;9(1):80

11. Chen $Y$, Pei $H$, Chang $Y$, Chen $M$, Wang $H$, Xie $H$, et al. The impact of endometrioma and laparoscopic cystectomy on ovarian reserve and the exploration of related factors assessed by serum anti-Mullerian hormone: a prospective cohort study. J Ovarian Res. 2014;7(1):108.

12. Fanchin R, Schonäuer LM, Righini C, Frydman N, Frydman R, Taieb J. Serum anti-Mullerian hormone dynamics during controlled ovarian hyperstimulation. Hum Reprod. 2003;18(2):328-32.

13. Pabuccu R, Onalan G, Goktolga U, Kucuk T, Orhon E, Ceyhan T. Aspiration of ovarian endometriomas before intracytoplasmic sperm injection. Fertil Steril. 2004;82(3):705-11.

14. Bendifallah S, Roman H, Mathieu DE, Touleimat S, Cohen J, Darai E, et al. Colorectal endometriosis-associated infertility: should surgery precede ART? Fertil Steril. 2017:108(3):525-31.

15. Geber S, Ferreira DP, Prates LFVS, Sales L, Sampaio M. Effects of previous ovarian surgery for endometriosis on the outcome of assisted reproduction treatment. Reprod BioMed Online. 2002;5(2):162-6.
16. Loo TC, Lin MY, Chen SH, Chung MT, Tang HH, Lin LY, et al. Endometrioma undergoing laparoscopic ovarian cystectomy: its influence on the outcome of in vitro fertilization and embryo transfer (IVF-ET). J Assist Reprod Genet. 2005:22(9-10):329-33.

17. Suzuki T, Izumi S, Matsubayashi H, Awaji H, Yoshikata K, Makino T. Impact of ovarian endometrioma on oocytes and pregnancy outcome in in vitro fertilization. Fertil Steril. 2005;83(4):908-13.

18. Alazemi M, Killick SR, Duffy S, Pye C, Refaat B, Hill N, et al. Multi-marker assessment of ovarian reserve predicts oocyte yield after ovulation induction. Hum Reprod. 2011;26(2):414-22.

19. Koji N, Shirei O, Rieko K, Kana S, Megumu I, Takashi H, et al. Impact of laparoscopic cystectomy on fecundity of infertility patients with ovarian endometrioma. J Obstet Gynaecol Res. 2007;33(5):671-6.

20. Canis M, Pouly JL, Tamburro S, Mage G, Wattiez A, Bruhat MA. Ovarian response during IVF-embryo transfer cycles after laparoscopic ovarian cystectomy for endometriotic cysts of $>3 \mathrm{~cm}$ in diameter. Hum Reprod. 2001;16(12):2583-6.

21. Wong BC, Gillman NC, Oehninger S, Gibbons WE, Stadtmauer LA. Results of in vitro fertilization in patients with endometriomas: is surgical removal beneficial? Am J Obstet Gynecol. 2004;191(2):597-605.

22. Nelson SM, Stamatina I, Richard F, Richard A, Alex MC, Claudia-Martina M. Reference range for the antimüllerian hormone generation II assay: a population study of 10,984 women, with comparison to the established diagnostics systems laboratory nomogram. Fertil Steril. 2014;101(2):523-9.

23. Younis JS, Shapso N, Fleming R, Ben-Shlomo I, Izhaki I. Impact of unilateral versus bilateral ovarian endometriotic cystectomy on ovarian reserve: a systematic review and meta-analysis. Hum Reprod Update. 2019;25(3):37591.

24. Mehdizadeh KA, Chaichian S, Ariana S, Fazaeli M, Moradi Y, Rashidi M, et al. The impact of laparoscopic cystectomy on ovarian reserve in patients with unilateral and bilateral endometrioma. Int J Gynaecol Obstet. 2017;136(2): 200.

25. Wang $T$, Ke ZH, Song $Y$, Chen LT, Chen XJ, Feng $C$, et al. Identification of a mutation in GDF9 as a novel cause of diminished ovarian reserve in young women. Hum Reprod. 2013;28(9):2473-81.

26. Pascale MP, Véronique FLT, Catherine M, Charles M, Sophie L, Marie-Claire $M$, et al. Molecular characterization of corona radiata cells from patients with diminished ovarian reserve using microarray and microfluidic-based gene expression profiling. Hum Rreprod. 2012;27(3):829-43.

27. Steiner AZ, Pritchard D, Stanczyk FZ, et al. Association between biomarkers of ovarian reserve and infertility among older women of reproductive age. JAMA. 2017;318(14):1367-76

28. Somigliana E, Ragni G, Infantino M, Benedetti F, Arnoldi M, Crosignani PG. Does laparoscopic removal of nonendometriotic benign ovarian cysts affect ovarian reserve? Acta Obstet Gyn Scan. 2011;85(1):74-7.

29. Garciavelasco JA, Somigliana E. Management of endometriomas in women requiring IVF: to touch or not to touch. Hum Rreprod. 2009:24(3):496-501.

30. Ata B, Turkgeldi E, Seyhan A, Urman B. Effect of hemostatic method on ovarian reserve following laparoscopic endometrioma excision; comparison of suture, hemostatic sealant and bipolar dessication; a systematic review and meta-analysis. J Minim Invas Gyn. 2015;22(3):363-72.

31. Dong-Yun L, Na YK, Min JK, Byung-Koo Y, Dooseok C. Effects of laparoscopic surgery on serum anti-Müllerian hormone levels in reproductive-aged women with endometrioma. Gynecol Endocrinol. 2011;27(10):733-6.

32. Edgardo S, Nicola B, Laura B, Paola V, Paolo V, Luigi F. Surgical excision of endometriomas and ovarian reserve: a systematic review on serum antimüllerian hormone level modifications. Fertil Steril. 2012:98(6):1531-8.

33. Benaglia L, Somigliana E, Vercellini $P$, Benedetti F, lemmello R, Vighi V, et al. The impact of IVF procedures on endometriosis recurrence. Eur J Obstet Gynecol Reprod Biol. 2010;148(1):49-52.

34. Park HJ, Kim H, Lee GH, Yoon TK, Lee WS. Could surgical management improve the IVF outcomes in infertile women with endometrioma?: a review. Obstet Gynecol Sci. 2019;62(1):1-10

35. Guo YH, Lu N, Zhang Y, Su YC, Wang Y, Zhang YL, et al. Comparative study on the pregnancy outcomes of in vitro fertilization-embryo transfer between long-acting gonadotropin-releasing hormone agonist combined with transvaginal ultrasound-guided cyst aspiration and long-acting gonadotropin-releasing hormone agonist alone. Contemp Clin Trials. 2012; 33(6):1206-10.

36. Lebovic DI, Mueller MD, Taylor RN Immunobiology of endometriosis. Fertil Steril. $2001 ; 75(1): 1-10$ 
37. Tamura H, Takasaki A, Nakamura Y, Numa F, Sugino N. A pilot study to search possible mechanisms of ultralong gonadotropin-releasing hormone agonist therapy in IVF-ET patients with endometriosis. J Ovarian Res. 2014; 7(1):100.

38. María-José GT, Pedro A, Adolfo C, Irene V. Embryotoxicity of peritoneal fluid in women with endometriosis. Its relation with cytokines and lymphocyte populations. Hum Rreprod. 2002;17(3):777.

39. Wu MY, Chen SU, Chao KH, Chen CD, Yang YS, Hong NH. Mouse embryo toxicity of IL-6 in peritoneal fluids from women with or without endometriosis. Acta Obstet Gynecol Scand. 2001;80(1):7-11.

40. Xu Y, Zhang YS, Zhu DY, Zhai XH, Wu FX, Wang AC. Influence of GnRH antagonist in reproductive women on in vitro fertilization and embryo transfer in fresh cycles. Biomed Rep. 2019;10(2):113-8.

41. Meldrum DR, Chang JR, RJ G, Linda LC, Balasch J, Barbieri RL, et al. Role of decreased androgens in the ovarian response to stimulation in ;older women. Fertil Steril. 2013;99(1):5-11.

42. Meldrum DR, Casper RF, Diez-Juan A, Simon C, Domar AD, Frydman R. Aging and the environment affect gamete and embryo potential: can we intervene? Fertil Steril. 2016;105(3):548-59.

43. Harlev A, Gupta S, Agarwal A. Targeting oxidative stress to treat endometriosis. Expert Opin Ther Targets. 2015;19(11):1447-64.

44. Rosa VLL, Barra F, Chiofalo B, et al. An overview on the relationship between endometriosis and infertility: the impact on sexuality and psychological well-being. J Psychosom Obstet Gynecol. 2019;30:1-5.

\section{Publisher's Note}

Springer Nature remains neutral with regard to jurisdictional claims in published maps and institutional affiliations.

Ready to submit your research? Choose BMC and benefit from:

- fast, convenient online submission

- thorough peer review by experienced researchers in your field

- rapid publication on acceptance

- support for research data, including large and complex data types

- gold Open Access which fosters wider collaboration and increased citations

- maximum visibility for your research: over $100 \mathrm{M}$ website views per year

At $\mathrm{BMC}$, research is always in progress.

Learn more biomedcentral.com/submissions 